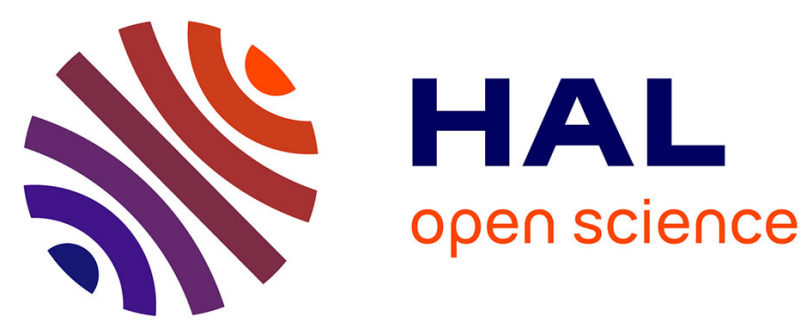

\title{
Revealing the Structure of a Granular Medium through Ballistic Sound Propagation
}

\author{
S. Lherminier, R. Planet, Grégory Simon, L. Vanel, O. Ramos
}

\section{To cite this version:}

S. Lherminier, R. Planet, Grégory Simon, L. Vanel, O. Ramos. Revealing the Structure of a Granular Medium through Ballistic Sound Propagation. Physical Review Letters, 2014, 113 (9), pp.098001. 10.1103/PhysRevLett.113.098001 . hal-01727331v1

\section{HAL Id: hal-01727331 https://univ-lyon1.hal.science/hal-01727331v1}

Submitted on 12 Mar 2018 (v1), last revised 16 Mar 2018 (v2)

HAL is a multi-disciplinary open access archive for the deposit and dissemination of scientific research documents, whether they are published or not. The documents may come from teaching and research institutions in France or abroad, or from public or private research centers.
L'archive ouverte pluridisciplinaire HAL, est destinée au dépôt et à la diffusion de documents scientifiques de niveau recherche, publiés ou non, émanant des établissements d'enseignement et de recherche français ou étrangers, des laboratoires publics ou privés. 


\title{
Revealing the Structure of a Granular Medium through Ballistic Sound Propagation
}

\author{
S. Lherminier, R. Planet, ${ }^{*}$ G. Simon, L. Vanel, and O. Ramos \\ Institut Lumière Matière, UMR5306 Université Lyon 1-CNRS, Université de Lyon, 69622 Villeurbanne, France
}

(Received 15 December 2013; published 28 August 2014)

\begin{abstract}
We study the propagation of sound through a bidimensional granular medium consisting of photoelastic disks, which are packed into different crystalline and disordered structures. Acoustic sensors placed at the boundaries of the system capture the acoustic signal produced by a local and well-controlled mechanical excitation. By compressing the system, we find that the speed of the ballistic part of the acoustic wave behaves as a power law of the applied force with both exponent and prefactor sensitive to the internal geometry of the contact network. This information, which we are able to link to the force-deformation relation of single grains under different contact geometries, provides enough information to reveal the structure of the granular medium.
\end{abstract}

DOI: 10.1103/PhysRevLett.113.098001

PACS numbers: 45.70.-n, 43.25.+y, 46.40.Cd, 64.70.qj

In addition to its practical and industrial relevance, granular materials have lately gained a lot of attention from the scientific community [1], as they have become simplified models of complex phenomena: jamming transition [2-4], self-organized criticality [5], earthquake dynamics [6,7], etc. Being able to monitor the structure of the system in a tabletop experiment is one of the advantages of these simplified models, and (especially in the case of earthquakes) acoustics is often the most appropriated tool of analysis. However, granular materials are still reticent to leave clear fingerprints on acoustic waves [8-10]. This "misbehavior" is directly related to the fact that, in a granular medium, loads are transferred through contact mechanisms between neighboring particles creating force chains [11]. These chains are responsible for a huge degree of heterogeneity inside the system [12], which increases the dispersion of the signal. It is also known that acoustic waves favor speed and amplitude along force chains [13], and making the scenario more complex, contacts can activate and deactivate during the passage of the acoustic wave [14,15], amplifying its attenuation.

In this Letter, we show that valuable information of the internal structure of a compressed granular medium can be extracted by studying the ballistic (i.e., nondispersive) part of the sound wave. This contrasts with techniques based on the multiple scattering of acoustic waves that have received special attention in recent years [16-20]. Unlike the diffusive part of the signal, the ballistic part provides a more direct and simpler analysis of the granular structure. In strained granular materials, the speed of sound $c$ has a nonlinear behavior with the applied force $F$ as $c=K F^{\alpha}$ $[21,22]$. By studying the compression of granular materials with different crystalline structures, we found that the exponent $\alpha$ depends on the contact number of the grains, while the prefactor $K$ brings information about the anisotropy of the medium. When compressing disordered structures, we can observe different scalings, which indicates a change in the internal structure of the medium. Being able not only to detect a change in the structure of the granular system but also to recognize its nature is of great interest when trying to predict the abrupt events denominated avalanches [23-25], earthquakes [6], or unjamming events $[3,26]$, according to the subject of the study.

The experimental setup consists of a monolayer of disks confined into a rectangular cell placed vertically (see Fig. 1). This cell has an initial size of $162 \times 96 \mathrm{~mm}^{2}$ $(W \times H)$ and $3.6 \mathrm{~mm}$ thickness. The surrounding walls are built in the same material as the grains. The bottom and lateral walls are fixed, while the upper one is mobile allowing the compression of the granular medium. In order to avoid three-dimensional (3D) effects, the cell is confined between two acrylic plates. The whole setup is held in an external metallic frame. We use cylindrical grains of $6.4 \mathrm{~mm}$ diameter $(d)$ and $3.5 \mathrm{~mm}$ thickness $(L)$. These disks are made of Durus White 430 and have been generated in a Objet30 3D printer. Profile measurements of the surface roughness of the printed grains give a

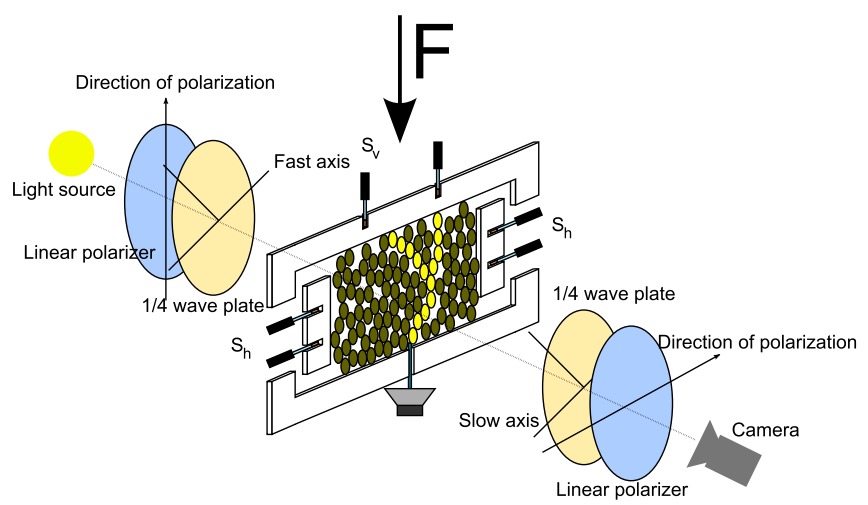

FIG. 1 (color online). Sketch of the experimental setup. $S_{v}$ and $S_{h}$ are acoustic sensors placed on the top wall and lateral walls, respectively. 
peak-to-peak maximum value of $25 \mu \mathrm{m}$ on the curved side of the cylinders. The zero-frequency Young modulus of Durus material is $E \simeq 100 \mathrm{MPa}$. The translucent and birefringent character of the grains allows the visualization of the stress inside the disks when placing the experimental setup between two circular polarizers (Fig. 2). We take images of the experiment with a Nikon D700 camera $(0.045 \mathrm{~mm} /$ pixel resolution).

In order to set the structure of the packing, we have designed specific boundary conditions that consists of a row of half grains separated by a specific gap between them and fixed to the bottom wall [27]. We have studied three different crystalline structures (Fig. 2): squared at $45^{\circ}$ (S45), squared at $90^{\circ}$ (S90), hexagonal at $60^{\circ}$ (H60), and different disordered structures (D). For comparison, we have also performed an experiment in a one-dimensional configuration $(W=d[22])$. The disordered structures are obtained by pouring the grains randomly between the acrylic plates. Figure 2(d) shows the disposition of the grains (a video in the Supplemental Material [28] shows the contraction of the system).

The granular medium is excited mechanically by a thin metallic rod connected to a loudspeaker (see Fig. 1). The rod moves freely through a hole at the center of the bottom wall and hits one grain. This generates an acoustic wave of about $4 \mathrm{kHz}$ frequency with a pressure amplitude much smaller than the confining pressure to ensure the propagation of linear acoustic waves. For this frequency, the speed of sound in Durus is $\simeq 1200 \mathrm{~m} / \mathrm{s}$. The acoustic signal travels across the medium (see a video of the wave propagation in the Supplemental Material [28]) and is recorded by six acoustic sensors (CTS Valpey Corporation VP-1.5) that are placed on the lateral and upper walls of the cell. Because of the experimental configuration, we are only measuring compressional waves ( $P$ waves), which are

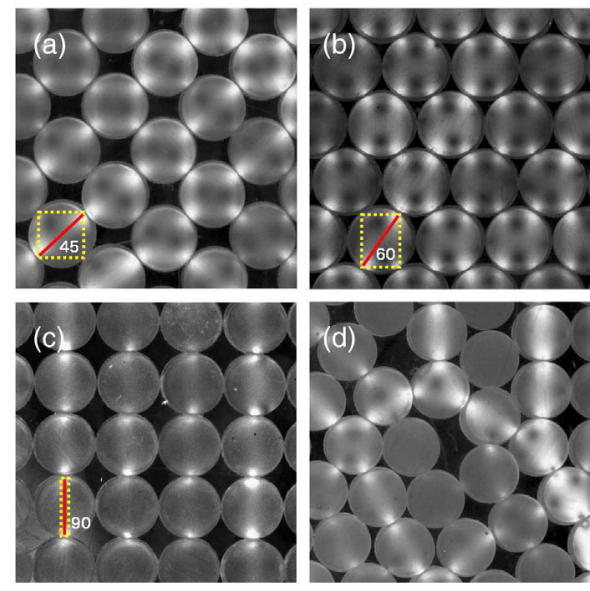

FIG. 2 (color online). Detail of the granular monolayer for the four different geometries studied: (a) S45, (b) H60, (c) S90, and (d) D. By placing the setup between two crossed circular polarizers, it is possible to identify the contacts. the fastest and the first to be recorded. In order to induce a deformation in the granular system, we set a jack between the upper wall of the cell and the metallic frame. This jack is connected to a force sensor (Interface, SML-300 with a stiffness of $1.75 \times 10^{7} \mathrm{~N} / \mathrm{m}$ ) that measures the applied force over the grains.

First, we apply a given deformation over the system. Then, we excite the granular medium by sending an electrical signal to the loudspeaker. The emitted acoustic signal and the applied force are recorded continuously by an NI-USB-6366 card at a frequency of $2 \mathrm{MHz}$ during $1.1 \mathrm{~s}$ (150 ms before and $950 \mathrm{~ms}$ after the emission). We have repeated the experiments with the same conditions to check the reproducibility of the results. Finally, for each acoustic excitation, we record the image of the whole system, and a simple routine detects the number of contacts of each grain. In order to extract the time of flight, we perform a spectral analysis of the recorded signal [29]. This method provides more accurate results than a direct thresholding since it takes into account all the frequencies and detects the quickest one (independent of dispersive effects).

Figure 3 shows the speed of sound as a function of the applied force over the system. As expected, we observe $c \sim F^{\alpha}$. We point out the existence of two different sets of results. For experiments performed on structures S45 and H60 (that have 4 contacts per grain), we obtain $\alpha=0.16 \pm$ 0.03 while for S90 (2 contacts per grain) we get $\alpha=0.50 \pm 0.06$, that is, the same scaling observed for the one-dimensional case [inset in Fig. 3(c)]. The result for
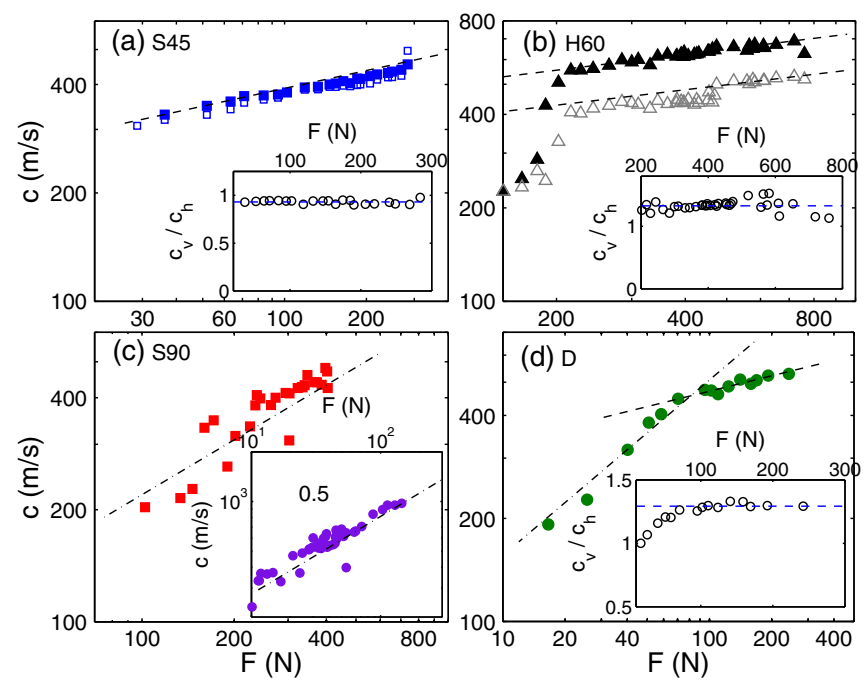

FIG. 3 (color online). Scaling of the sound speed as a function of the applied force for the different structures studied. Filled symbols and open symbols correspond to measurements at the top wall $\left(c_{v}\right)$ and lateral walls $\left(c_{h}\right)$, respectively. The insets in (a), (b), and (d) show $c_{v} / c_{h}$. The inset in (c) corresponds to the speed of sound through a one-dimensional chain of cylinders. The straight lines in the figures correspond to guides to the eye for two different power-law behaviors: (- - -) correspond to $F^{0.16}$ and $(-\cdot-)$ to $F^{0.5}$. 
the one-dimensional chain of cylinders differs from the one reported in Ref. [22]. It is important to point out the existence of a preliminary regime at low $F$ (evident in structure H60) that corresponds to the progressive activation of contacts in the granular lattice. A special case is the disordered configuration $\mathrm{D}$ where we observe the two scaling behaviors. For low loadings, we observe the 0.5 exponent, and at larger forcing we recover the 0.16 exponent. In the case shown in Fig. 3(d), the crossover that separates both regimes appears at $80 \mathrm{~N}$. This crossover does not appear at the same applied force for different experiments, and it is very dependent on the initial configuration of the specific experiment, which is a common issue in granular media [1].

The propagation of sound in granular materials is governed by the contact mechanics of its grains [30]. By analogy with the discrete model of phonons in a solid [31], we consider that the sound speed $c$ in a chain of grains of stiffness $\kappa$, mass $m$, and distance between contacts $d_{c}$ is

$$
c=\sqrt{\kappa / m} d_{c} \cos \left(\pi d_{c} / \lambda\right) \simeq \sqrt{\kappa / m} d_{c}
$$

in the regime where the wavelength $\lambda$ is large compared to the grain size [32]. A similar scaling was already proposed by previous authors (see Ref. [33] and references therein). In this case, $\kappa$ can be written as $d f / d \delta$, where $f$ corresponds to the contact force between grains, and $\delta$ is the deformation [34]. In order to recreate the contact mechanics of the experiments shown in Fig. 3, we have performed experiments where a single grain is compressed between two flat plates and between two $\pi / 2$ wedges (see Fig. 4), made of Duralumin, that provide 2 and 4 flat contacts to the grain, respectively. Figure 4 shows the force-deformation curves for a single cylindrical grain in the two different contact geometries. In the 2 contacts geometry, the force-deformation curve shows a power-law regime with

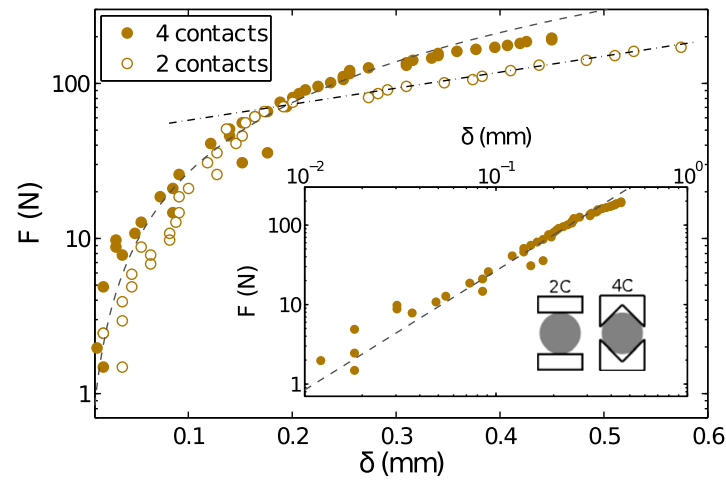

FIG. 4 (color online). Measured force-deformation relation for a single grain in a 2 contacts geometry $(2 \mathrm{C})$ and in a 4 contacts geometry (4C). The power law $f \sim \delta^{3 / 2}$ is represented by discontinuous lines while the exponential law $f \sim e^{\delta}$ is represented by a dot-dashed line; $\delta$ is the total deformation of the compressed grain $\left(\delta=2 \delta_{1}\right)$. an exponent $\beta \simeq 3 / 2$ for small forces, with a crossover to an exponential behavior for large forces. In the 4 contacts geometry, the power-law relation is more robust and spans a larger range of deformations. In this case, we extract the value of the exponent $\beta=1.5 \pm 0.3$ (see inset in Fig. 4). A very similar value for the power-law exponent has been already reported for viscoelastic cylinders, and deviation from the expected value (Supplemental Material [28]) has been attributed to the role of asperities (see the Supplemental Material of Ref. [4]). Grains made with different materials follow the same behavior (Supplemental Material [28]), which indicates that the elastic properties of the cylinders prevail in this forcedeformation relation. With this information and using $\kappa=d f / d \delta$ and $c \sim \kappa^{1 / 2}$, we can calculate the $\alpha$ exponent (assuming $F \sim f$ ). If $f \sim \delta^{\beta}$ with an exponent $\beta \simeq 3 / 2$, then $c \sim F^{(\beta-1) / 2 \beta} \sim F^{\alpha}$ with $\alpha \simeq 1 / 6$, while a relation $f \sim e^{\delta}$ will give $c \sim \sqrt{F}$, then $\alpha=1 / 2$. As the distribution of forces is highly inhomogeneous (even in a regular structure [12]), and force chains dominate the dynamics, in the two contacts geometry we do not notice the low forces regime with a power-law exponent $1 / 6$ and only detect the exponent $1 / 2$, associated with the exponential part in the force-deformation relation of Fig. 4. This also explains the exponent at low force observed in Fig. 3(d). In the case of 4 contacts, the normal force per contact for a given applied force per grain will be significantly lower than in the 2 contacts case, which increases the possibility to observe the power-law regime in the force-deformation relation leading to the power-law exponent $1 / 6$ in Fig. 3 . The inverse transition observed in Fig. 3(d) compared to the transition observed in Fig. 4 when increasing the applied force corresponds to the progressive shift from the large force exponential regime for a 2 contacts configuration to the large force but still power-law regime for a 4 contacts configuration that can be observed at the same applied force. This evolution of the grain coordination number is discussed in more detail below. From that perspective, the experimental observations plotted in Fig. 3 are in perfect agreement with the force-deformation relations of single grains. Also, from Fig. 4 we find that the effective stiffness varies between $3 \times 10^{5}$ and $2 \times 10^{6} \mathrm{~N} / \mathrm{m}$. From Eq. (1) we get $c \simeq 370-860 \mathrm{~m} / \mathrm{s}$, which corresponds rather well to the measured range of sound speeds.

This analysis reinforce the fact that the exponent $\alpha$ appears to be directly related with the coordination number of the grains. To confirm this in the case of the disordered configuration, Fig. 5 shows the number of grains that have 2,3 , and 4 contacts, as a function of the applied force. This plot shows that at $80 \mathrm{~N}$ the number of grains with 4 contacts start to increase from $0 \%$ to $20 \%$. This crossover coincides with the change in the $\alpha$ exponent, indicating that the change on the scaling of $c$ with $F$ corresponds to a change in the coordination number of the grains involved in the path of the sound wave. 


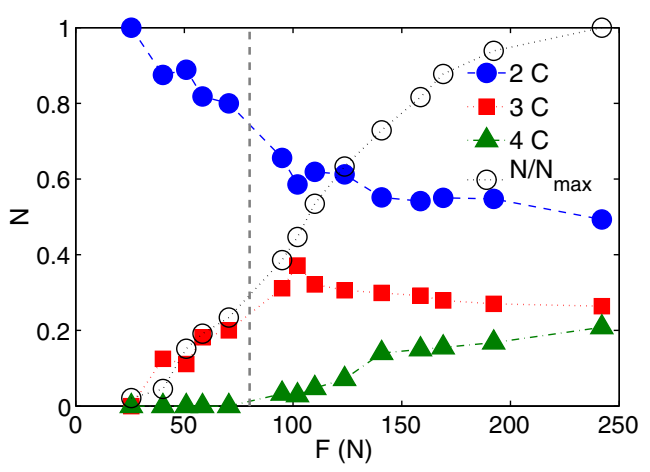

FIG. 5 (color online). Fraction of grains with 2, 3, or 4 contacts in the experiment carried out with D configuration (same as Fig. 3). The vertical dashed line marks the crossover between the two power-law regions observed in Fig. 3. The total number of contacts normalized by its value at the highest force shows a continuous trend without any abrupt change of behavior at $80 \mathrm{~N}$.

Figure 3(b) also shows the existence of a vertical shift in the $c$ vs $F$ curve between sensors placed in the top part and the lateral walls. This effect is clear for the case of H60 and does not occur in the S45 configuration. The insets of Figs. 3(a), 3(b), and 3(d) display the ratios between the speed of sound measured on the top part $c_{v}$ and the side walls $c_{h}$ (the values of $c_{h}$ are negligible compared to $c_{v}$ in the case of S90).

Since the sound propagation is governed by the contact mechanics between grains, the absolute value of the recorded signal will be also affected by the relative orientation of the acoustic source and the sensors, but more important, by the anisotropy of the contact distribution for single grains. In a regular configuration, the distances $d_{c}$ between contacts in the vertical and horizontal directions are proportional to $d \sin \theta$ and $d \cos \theta$, respectively, where $\theta$ is the angle between neighbors at different rows (see Fig. 2). Then, from Eq. (1), the scaling of the vertical and horizontal sound speed are $c_{y} \sim \sin \theta$ and $c_{x} \sim \cos \theta$. Finally, the speed of sound recorded by a sensor placed at a horizontal angle $\eta$ with respect to the source follows: $c_{\eta} \sim \sin \theta \sin \eta+\cos \theta \cos \eta$. Since the angles of the top and lower lateral sensors are $\eta_{v} \sim 70^{\circ}$ and $\eta_{h} \sim 20^{\circ}$, respectively, the ratio of the velocities correspond to 1 for $\mathrm{S} 45\left(\theta=45^{\circ}\right)$ and 1.3 for $\mathrm{H} 60$ $\left(\theta=60^{\circ}\right)$, which is consistent with the measured values plotted in the inset of Fig. 3 where we observe 0.93 and 1.33 for $\mathrm{S} 45$ and $\mathrm{H} 60$, respectively. The ratio $c_{v} / c_{h}$ for the disordered configuration [Fig. 3(d)] is evolving towards a more stable geometry ("H60-like") at the crossover force $80 \mathrm{~N}$.

Finally, we are able to identify the "main" coordination number of a granular matter with an a priori unknown structure by studying the scaling of the sound speed as a function of applied loads (we use the word "main" to refer to the chain that supports a stronger load favoring the propagation of the sound wave). This is due to a different behavior of the force-deformation response of single grains under different contact geometries. In contrast with previous works, we observe that the relation between the force and deformation of two cylindrical grains in contact with parallel axis crosses over from a power law to an exponential law when increasing the applied load. This last result is supported by the observation of the scaling $c \sim F^{1 / 2}$. The relation of force and deformation for the present contact geometry with purely elastic cylinders has the form [30]

$$
\delta_{1}=\frac{1-\nu^{2}}{\pi E} \frac{f}{L}\left[\ln \left(\frac{4 \pi E R L}{\left(1-\nu^{2}\right) f}\right)-1\right],
$$

where $\delta_{1}$ is the compressed distance due to a single contact, $\nu$ corresponds to the Poisson's ratio (typically $\nu \simeq 0.4$ for polymeric materials [35]), and $R$ correspond to cylinder radius. This equation can be written in an adimensional form by defining $\tilde{f}=f\left(1-\nu^{2}\right) /(4 \pi E R L)$ and $\tilde{\delta}=\delta_{1} /$ $(4 R)$. So, if we perform a Taylor expansion of this relation around an imposed force $f_{0}$, the resulting expansion can be written as

$$
\tilde{\delta}=-\left[\tilde{f}_{0}+2 \tilde{f}_{0} \ln \tilde{f}_{0}\right]-\left(3+\ln \tilde{f}_{0}\right) \epsilon+\tilde{f}_{0} \ln \left(\tilde{f}_{0}+\epsilon\right),
$$

where $\epsilon=\tilde{f}-\tilde{f}_{0}$ (see the Supplemental Material [28]). Equation (2) shows two different regimes: for low applied forces $f_{0}$, the term $\left(3+\ln \tilde{f}_{0}\right) \tilde{f}$ dominates and that would correspond to a linear behavior for cylinders $f \sim \delta$ (however, this is true only for extremely low forces and can be approximated as a power law at larger ones, Supplemental Material [28]), whereas for larger $f_{0}$, the term $\tilde{f}_{0} \ln \tilde{f}$ dominates and that would correspond to an exponential law $f \sim e^{\delta}$. The two regimes are separated by a crossover $f_{c}$ given by the weight of the prefactors $f_{c}=e^{-4} 4 \pi E R L /(1-$ $\nu^{2}$ ) (Supplemental Material [28]). Even though $f_{c}$ does not match perfectly with the experimental results, Eq. (2) gives an explanation for the observation of an exponential regime with purely elastic considerations. We believe this discrepancy in $f_{c}$ lies in the inherent viscoelasticity of polymeric materials and in a finite length of the cylinders. We also would like to point out that Eq. (2) is derived from a model defined for small deformation. However, the behavior of the force-deformation relation given by the model for a range of forces $(\delta / R)>0.1$ predicts an exponential law, which is qualitatively what is observed in our experimental results (Supplemental Material [28]). Smaller values in $\delta / R$ may be a reason why the transition was not observed in Ref. [22]. This transition may correspond to the global mechanical response that changes the force-deformation relation from a power law to an exponential one in a smooth contact between cylinders. The exponent value 1.5 in the force-deformation relation may correspond to the local effect of spherical-like asperities, which can play a role for 
deformations up to 50 times the amplitude of the roughness [22]. However, this effect does not seem to affect the transition to an exponential behavior, which may correspond to a global deformation of the grain and is reached before a change in the exponent value found in Ref. [22] and linked to the roughness of the grains.

To sum up, we have performed an experiment with photoelastic disks where by analyzing the ballistic part of an acoustic signal generated in the system, we are able to get information about the average coordination number of the grains involved in the acoustic path. This information is contained in the exponent of the speed-force relation, being $\alpha \simeq 0.5$ for 2 contacts and $\alpha \simeq 0.16$ for 4 contacts. The quotient $c_{v} / c_{h}$ brings information about the anisotropy of the system. With this information, it is possible to reveal the structure of the granular packing in a neighborhood of a line between the source of the acoustic signal and the sensor. We have also shown that this speed-force relation is a consequence of the force-deformation relation of single grains. Being able to get information about the internal structure of a compressed granular medium with acoustic measurements is of great practical interest. Stability analysis based on the contact topology of the grains [36], avalanche prediction, and simplified models of earthquakes are a few examples.

We thank K. Daniels, P. Jop, and V. Tournat for fruitful discussions. This work is supported by the AXA Research Fund.

*ramon.planet@gmail.com

[1] H. M. Jaeger, S. R. Nagel, and R. P. Behringer, Rev. Mod. Phys. 68, 1259 (1996).

[2] P. Richard, M. Nicodemi, R. Delannay, P. Ribiere, and D. Bideau, Nat. Mater. 4, 121 (2005).

[3] M. Wyart, L. E. Silbert, S. R. Nagel, and T. A. Witten, Phys. Rev. E 72, 051306 (2005).

[4] T. S. Majmudar, M. Sperl, S. Luding, and R. P. Behringer, Phys. Rev. Lett. 98, 058001 (2007).

[5] P. Bak, C. Tang, and K. Wiesenfeld, Phys. Rev. Lett. 59, 381 (1987).

[6] P. A. Johnson, H. Savage, M. Knuth, J. Gomberg, and C. Marone, Nature (London) 451, 57 (2008).

[7] F. Brenguier, M. Campillo, C. Hadziioannou, N. M. Shapiro, R. M. Nadeau, and E. Larose, Science 321, 1478 (2008).

[8] C.-h. Liu and S. R. Nagel, Phys. Rev. Lett. 68, 2301 (1992).

[9] C.-h. Liu and S. R. Nagel, Phys. Rev. B 48, 15646 (1993).

[10] X. Jia, C. Caroli, and B. Velicky, Phys. Rev. Lett. 82, 1863 (1999).

[11] T. S. Majmudar and R. P. Behringer, Nature (London) 435 , 1079 (2005).

[12] D. L. Blair, N. W. Mueggenburg, A. H. Marshall, H. M. Jaeger, and S. R. Nagel, Phys. Rev. E 63, 041304 (2001).
[13] E. T. Owens and K. E. Daniels, Europhys. Lett. 94, 54005 (2011).

[14] C. F. Schreck, T. Bertrand, C. S. O'Hern, and M. D. Shattuck, Phys. Rev. Lett. 107, 078301 (2011).

[15] B. Gilles and C. Coste, Phys. Rev. Lett. 90, 174302 (2003).

[16] R. L. Weaver and O. I. Lobkis, Phys. Rev. Lett. 87, 134301 (2001).

[17] M. L. Cowan, I. P. Jones, J. H. Page, and D. A. Weitz, Phys. Rev. E 65, 066605 (2002).

[18] E. Larose, J. De Rosny, L. Margerin, D. Anache, P. Gouedard, M. Campillo, and B. van Tiggelen, Phys. Rev. E 73, 016609 (2006)

[19] K. Wapenaar, F. Broggini, E. Slob, and R. Snieder, Phys. Rev. Lett. 110, 084301 (2013).

[20] S. Palus, Physics 6, 21 (2013).

[21] J. D. Goddard, Proc. R. Soc. A 430, 105 (1990).

[22] G. Huillard, X. Noblin, and J. Rajchenbach, Phys. Rev. E 84, 016602 (2011).

[23] O. Ramos, E. Altshuler, and K. J. Måløy, Phys. Rev. Lett. 102, 078701 (2009).

[24] S. K. de Richter, V. Y. Zaitsev, P. Richard, R. Delannay, G. L. Caër, and V. Tournat, J. Stat. Mech. (2010) P11023.

[25] T. Shinbrot, N. H. Kim, and N. N. Thyagu, Proc. Natl. Acad. Sci. U.S.A. 109, 10806 (2012).

[26] T. Shinbrot, Nat. Phys. 9, 263 (2013).

[27] E. Altshuler, O. Ramos, C. Martínez, L. E. Flores, and C. Noda, Phys. Rev. Lett. 86, 5490 (2001).

[28] See Supplemental Material at http://link.aps.org/ supplemental/10.1103/PhysRevLett.113.098001 for details.

[29] First, we normalize the acoustic signal $p(t)$ by its energy $P(t)=p(t) / \int_{0}^{1.1 \mathrm{~s}} p^{2}(t)$ in order to have comparable amplitude levels for all the sensors. Second, we perform the power spectrum over temporal windows of $64 \mu \mathrm{s}$ $S_{t_{i}}(f)=\widetilde{P}_{t_{i}}(f) \widetilde{P}_{t_{i}}(-f)$, where $\widetilde{P}_{t_{i}}(f)$ corresponds to the Fourier transform of $P(t)-\langle P(t)\rangle$ in the temporal window centered at $t_{i}$. Finally, we sum the spectral amplitudes over all the frequencies $A_{t_{i}}=\sum_{f} S_{t_{i}}(f)$. We consider that the acoustic pulse has arrived when $A_{t_{r}}>10^{-4}$ (threshold is independent of the sensor). So the speed of sound is obtained as $c=D_{\text {euc }} / \Delta t$, where $D_{\text {euc }}$ corresponds to the Euclidean distance between the source of the wave and the acoustic sensor, $\Delta t=t_{r}-t_{0}$, and $t_{0}$ corresponds to the impact time.

[30] K. Johnson, Contact Mechanics (Cambridge University Press, Cambridge, England, 1985).

[31] C. Kittel, Introduction to Solid State Physics, 6th ed. (John Wiley \& Sons, Inc., New York, 1986).

[32] This approximation is justified in our experiments since the smallest value of $\lambda \simeq 200 \mathrm{~m} / \mathrm{s} / 4 \mathrm{kHz} \simeq 0.05 \mathrm{~m}$ gives $\cos \left(\pi d_{c} / \lambda\right) \simeq 1$ with less than $10 \%$ error.

[33] B. Velický and C. Caroli, Phys. Rev. E 65, 021307 (2002).

[34] When referring to the stress applied over a granular medium we use $F$, and we keep $f$ for contact forces between grains.

[35] D. V. Rosato, N. R. Schott, and M. G. Rosato, Plastics Institute of America Plastics Engineering, Manufacturing \& Data Handbook (Springer, New York, 2001).

[36] R. Arévalo, I. Zuriguel, and D. Maza, Phys. Rev. E 81, 041302 (2010). 